\title{
The Effect of Melatonin on Peripheral Blood Cells during Total Body Irradiation in Rats ${ }^{1)}$
}

\author{
Mehmet Koc, ${ }^{*, a}$ Mehmet Emin BuyukoKuroglu, ${ }^{b}$ and Seyithan TAYSI ${ }^{c}$ \\ ${ }^{a}$ Department of Radiation Oncology, Medical School of Ataturk University; ${ }^{b}$ Department of Pharmacology, Medical \\ School of Ataturk Universituy; and ${ }^{c}$ Department of Biochemistry, Medical School of Ataturk University; 25240 Erzurum, \\ Turkey. Received January 18, 2002; accepted February 19, 2002
}

\begin{abstract}
Melatonin, has been reported to participate in the regulation of a number of important physiological and pathological process. It has also the ability to protect the genetic material of hematopoietic cells of mice from damaging effects of acute total body irradiation. The objective of this study was to the potential radioprotective effects of pharmacological doses of melatonin in total body irradiated rat's peripheral blood cells. Forty adult rats were divided into 4 equal groups. Group 1 received no melatonin or irradiation (control group), while group 2 received only melatonin $(5 \mathrm{mg} / \mathrm{kg}$, i.p.). Group 3 received only total body irradiation (RT) by $5 \mathrm{~Gy}$ of $\gamma$ irradiation only and group 4 received RT plus melatonin $(5 \mathrm{mg} / \mathrm{kg}$, i.p., $30 \mathrm{~min}$ before RT). An hour and a half following RT, blood samples were taken. Leukocytes and thrombocytes number and hemoglobin levels were measured in all groups. Five $\mathrm{mg} / \mathrm{kg}$ dose of melatonin significantly protected leukocytes and as well as thrombocytes number against $\gamma$ irradiation. There were no significant differences between Hb levels. Our results suggest that melatonin administration prior to irradiation prevented radiation damage on peripheral blood cells. Melatonin radioprotection is achieved by its ability as a scavenger for free radicals generated by ionizing radiation and acts probably as a growth factor, especially for granulocytes in bone marrow.
\end{abstract}

Key words melatonin; total body irradiation; radioprotection

Melatonin ( $N$-acetyl-5-methoxytryptamine) is a hormone with high activity, which is produced by the pineal gland. Melatonin, the most known powerful antioxidants, stimulates the glutathione peroxidase (GSH-Px) and can play a role in the prevention from the oxidative damage. ${ }^{2)}$ Maestroni et al. ${ }^{3)}$ reported that the pineal hormone melatonin could rescue hematopoesis from the toxic effect of cancer chemotherapeutic compounds in Lewis lung carcinoma-bearing mice. Some of the reports confirm the protective effect of melatonin in rats treated with myelotoxic drugs. In vitro, melatonin protected committed myeloid progenitors from cytotoxic drugs, and this effect was neutralized by anti-granulocyte/ macrophage colony-stimulating factor (GM-CSF) monoclonal antibody (mAb). ${ }^{3,4)}$ Vijayalaxmi et al. reported that human peripheral blood lymphocytes which were pretreated with melatonin in vitro and then exposed to gamma radiation in vitro exhibited a significantly reduced incidence of chromosomal aberrations (CA) and micronuclei (MN), as compared with similarly irradiated lymphocytes wich were not pretreated with melatonin. ${ }^{5)}$ Lissoni et al. ${ }^{6}$ ) found that melatonin acts as a growth factor, especially for granulocytes in bone marrow and suggested that melatonin releases IL-2 which stimulates bone marrow cells. Recently, Anwar et al. ${ }^{7}$ reported the potential protective effect of melatonin on bone marrow and lymphoid tissues of rats exposed to cytotoxic drugs.

Total body irradiation exposure may lead to bone marrow failure. During most courses of wide field radical radiotherapy covering substantial amounts of the bone marrow (e.g. mantle or inverted Y for Hodgkin's disease), the blood should be measured twice weekly. ${ }^{7)}$ The pineal gland and its main secretory product, melatonin, may have a role as a biologic clock in the regulation of both cortisol and peripheral blood cells. ${ }^{8)}$ However, there was not any study related to melatonin has protective effect on peripheral blood cells in whole body irradiated rats.
In this study, we investigated the possible acute radioprotective effect of melatonin in vivo given before irradiation on peripheral blood cells in total body irradiated rats.

\section{MATERIALS AND METHODS}

Forty adult albino Sprague-Dawley rats, weighing 190 \pm $20 \mathrm{~g}$ bred at Ataturk University Medical School, Department of Pharmacology Experimental Animal Laboratory were used. Rats were divided into 4 equal groups. Group 1 received no melatonin or irradiation (control group), while group 2 received only melatonin $(5 \mathrm{mg} / \mathrm{kg}$, i.p., Sigma Co.). Group 3 received only total body irradiation (RT) by 5 Gy of $\gamma$ irradiation only and group 4 received RT plus melatonin $(5 \mathrm{mg} / \mathrm{kg}$, i.p., $30 \mathrm{~min}$ before RT). $500 \mathrm{cGy}$ total body irradiation (single fraction) with a $182 \mathrm{cGy} / \mathrm{min}$ rate was used. Irradiation was performed using a cobalt-60 teletherapy unit (Picker-C 9, U.S.A.), by two anterior-posterior opposing fields of total body. The dose was calculated central axis at a depth of $2 \mathrm{~cm}$. All irradiated animals were anaesthesized with $60 \mathrm{mg} / \mathrm{kg}$ ketamin $\mathrm{HCl}$ (Parke-Devis, Turkey) before the irradiation. An hour and a half following RT, $2 \mathrm{ml}$ of blood samples were taken and peripheral blood cells were counted with an automatic counter (GEN-8 Hematology Analyser). Leukocytes and thrombocytes counts and hemoglobin $(\mathrm{Hb})$ levels were determined in all groups.

Statistical analysis was performed using the SPSS (Statistical Package for Social Science; Windows version 10.0) packed program. The statistical comparison of results has been performed by using paired samples Student's $t$-test.

\section{RESULTS AND DISCUSSION}

Hemopoietic tissue - mainly bone marrow and lymphoid tissue are highly radiosensitive. The most marked effects are on the parent (stem) cells of the leukocytes, lymphocytes and 
Table 1. Leukocyte and Thrombocyte Counts and $\mathrm{Hb}$ Levels in All Groups

\begin{tabular}{lccc}
\hline \hline \multicolumn{1}{c}{ Groups } & Leukocyte & Thrombocyte & Hemoglobin \\
\hline Control & $5383 \pm 337$ & $1279167 \pm 51293$ & $16.65 \pm 0.46$ \\
Melatonin & $6366 \pm 895$ & $1280167 \pm 114116$ & $16.33 \pm 0.80$ \\
Irradiation & $4387 \pm 328$ & $954000 \pm 74984$ & $16.35 \pm 0.68$ \\
Melatonin plus irradiation & $6116 \pm 630^{* \dagger}$ & $1176333 \pm 39652^{* *,+}$ & $16.75 \pm 0.53$
\end{tabular}

$* p<0.01, * * p<0.001$, with respect to irradiation, by Student's $t$-test. $\dagger p<0.05$, $\ddagger p<0.01$, with respect to control, by Student's $t$-test.

platelets. Red cells are much less radiosensitive, as their life cycle much longer. ${ }^{9)}$ As can be seen in Table 1, leukocytes and as well as thrombocytes counts were significantly protected with $5 \mathrm{mg} / \mathrm{kg}$ dose of melatonin against irradiation. But there was no significant difference between $\mathrm{Hb}$ levels. Moreover leukocyte count was significantly high in melatonin plus irradiation when compared to control group. Again, the most increasing leukocyte count was seen in only melatonin received group. Thrombocytes counts were lower in irradiation and melatonin plus irradiation groups than control. But melatonin plus irradiation group thrombocyte count was significantly higher than irradiation group. Therefore, melatonin protected the thrombocyte count against irradiation as in leukocyte count.

The radioprotective effect of melatonin was confirmed in vitro by Vijayalaxmi et al. ${ }^{5,10)}$ As assed by the induction of chromosomal aberrations (CAs) and micronuclei (MN) in cultured human blood lymphocytes. Confirmation of the same genetic test systems in vivo and in vitro has been reported by Vijayalaxmi et al. ${ }^{11)}$ They also reported that, whole body irradiated mice were pretreated with melatonin exhibited a significant and dose-dependent reduction in the observed incidence of micronuclei. They suggest that their data indicate melatonin has the ability to protect cells of mice from the damaging effect of acute total body irradiation. ${ }^{12}$ ) Badr et al. ${ }^{13)}$ reported that melatonin after irradiation did not protect againist induced CAs in spermatogonia. Their opinion melatonin after irradiation provides no radioprotective effect indicates that melatonin should be inside the cell at the time of exposure to radiation in order to confer protection. Knowing that ionizing radiation causes its harmful effect through generation of free radicals, ${ }^{14)}$ the melatonin principal mechanism of action for radioprotection, therefore, could be through its ability as a scavenger for free radicals. ${ }^{15)}$ The free radical-scavenging capacity of melatonin is mediated by electron donation. ${ }^{16)}$

Haldar et al. ${ }^{17)}$ reported that the pineal gland or its main hormone melatonin seems to have regulatory role in the proliferation of colony forming units for granulocytes and macrophages (CFU-GM) in rat bone marrow cell cultures. They claimed that regulatory role of the pineal gland and melatonin on the circaidian rhythm in colony formation of CFU-GM in intact and pinealectomized adult male rats. Akbulut et al. ${ }^{18)}$ found that the levels of leukocytes, neutrophils, and lymphocytes show daily changes in accordance with circadian rhythm in both healty controls and patients with breast cancer. They were also found serum melatonin, cortisol, and GM-CSF levels, and peripheral blood cell count showed significant circadian rhythms in healthy volunteers. They concluded GM-CSF, cortisol, and melatonin may have a role in the regulation of peripheral blood cell counts. Our study demonstrated that only-melatonin groups leukocyte counts significantly higher compared with sham control group $(p<0.001)$. Our results are in agreement with those obtained by Haldar et al. and Akbulut et al. In the light of literature, since red cells life cycle is about 4 months, this condition is normal. ${ }^{9)}$ Hsu et al. ${ }^{19)}$ reported that the peripheral blood count can be considered to be a biologically meaningful parameter to demonstrate the effect of radiation and radioprotection on a normal tissue which is critical survival. We thought that melatonin, has protective effect on peripheral blood cells, also could prevent intact tissues from effects of irradiation. Although our investigations might provide an experimental basis for the use of melatonin as a radioprotector of blood cells, its effect on other normal tissues such as the immune system, intestinal system and kidney should be further examined.

The data obtained in this study suggests that melatonin administration prevents damage inflicted by radiation when given prior to exposure to irradiation. Melatonin radioprotection is achieved by its ability as a scavenger for free radicals generated by ionizing radiation and probably acts as a growth factor, especially for granulocytes in bone marrow. Concomitant melatonin administration during radiotherapy may be effective in the treatment of irradiation-related myelosupression. Further studies are require in order to establish its possible mechanism(s) of radioprotective actions of melatonin on blood cells.

\section{REFERENCES AND NOTES}

1) Abstract was presented at The European Cancer Conference 11, Lisbon, October 21-25, 2001, EJC, 2001, 37(S6), p. 361.

2) Kaya H., Delibas N., Serteser M., Ulukaya E., Ozkaya O., Strahlenther and Onkol., 175, 285-288 (1999).

3) Maestroni G. J. M., Covacci A., Conti A., Cancer. Res., 54, 24292432 (1994a).

4) Maestroni G. J. M., Conti A., Lissoni P., Cancer. Res., 54, 4740—4743 (1994b).

5) Vijayalaxmi R. J. R., Sewerynek E., Poeggeler B., Leal B. Z., Meltz M. L., Radia. Res., 143, 102-106 (1995).

6) Lissoni P., Pittalis S., Brivio F., Tisi E., Rovelli F., Ardizzoia A., Barni S., Tancini G., Giudici G., Biondi A., Cancer., 71, 2076-2081 (1993).

7) Anwar M. M., Mahfouz H. A., Sayed A. S., Comp. Biochem. Physiol., 119, 493-591 (1998).

8) McNulty J. A., Relfson L. M., Fox L., Kus R. J., Handa G. B., Brain. Behav. Immunol., 4, 292-307 (1990).

9) Bomford C. K., Sherriff S. B., Kunkler I. H., Miller H. (eds.), “Textbook of Radiotherapy,” Longman Group UK Limited, 5th ed., 1993.

10) Vijayalaxmi R. J. R., Herman T. S., Meltz M. L., Mutat. Res., 346, 23-31 (1995).

11) Vijayalaxmi R. J. R., Herman T. S., Meltz M. L., Mutat. Res., 371, $221-228$ (1996).

12) Vijayalaxmi K. K., Reiter R. J., Leal B. Z., Meltz M. L., Mutat. Res., 351, 187-192 (1996).

13) Badr F. M., El Habit O. H. M., Harraz M. M., Mutat. Res., 444, 367372 (1999).

14) Scholes G., Br. J. Radiol., 56, $221-231$ (1983).

15) Reiter R. J., Tang L., Joaquin J., Garcia A. M., Hoyos D., Life Sci., 60, 2255-2271 (1997).

16) Hardeland R., Balzer I., Poeggeler B., Fuhrberg B., Uria H., Behrmann G., Wolf R., Meyer T. J., Reiter R. J., J. Pineal. Res., 18, 104-111 (1995).

17) Haldar C. D., Haussler D. G., J. Pineal. Res., 12, 79-83 (1992)

18) Akbulut H., Icli F., Büyükçelik A., Akbulut K. G., Demirci S., J. Pineal. Res., 26, 1-8 (1999).

19) Hsu H. Y., Yang J. J., Lian S. H., Ho Yh., Lin C. C., J. Ethnopharmacology., 54, 69-75 (1996). 